\title{
How dietary intake methodology is adapted for use in European immigrant population groups - a review
}

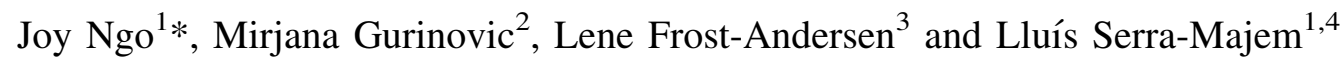 \\ ${ }^{1}$ Community Nutrition Research Centre of the Nutrition Research Foundation, University of Barcelona Science Park, Baldiri \\ Reixac 4, 08028 Barcelona, Spain \\ ${ }^{2}$ Department for Nutrition and Metabolism, Institute for Medical Research, University of Belgrade, Tadeusa Koscuska 1, Belgrade \\ 11000, Serbia \\ ${ }^{3}$ Department of Nutrition, University of Oslo, Postbox 1046 Blindern, 0316 Oslo, Norway \\ ${ }^{4}$ Department of Clinical Sciences, University of Las Palmas de Gran Canaria, PO Box 550, 35080 Las Palmas de Gran Canaria, \\ Spain
}

(Received 4 February 2009 - Revised 6 May 2009 - Accepted 1 June 2009)

Immigrants comprise a noteworthy segment of the European population whose numbers are increasing. Research on the dietary habits of immigrants is critical for correctly providing diet counselling and implementing effective interventions. The aim of the present study was to identify the presently used methods and adaptations required for measuring dietary intake in European immigrant groups. A comprehensive review strategy included a structured MEDLINE search, related references and key expert consultations. The review targeted adults from nonEuropean union (European union-15 countries) ethnic groups having the largest populations in Europe. As studies evaluating nutrient intake were scarce, papers evaluating intake at the level of foods were included. Forty-six papers were selected. Although Eastern Europe, Turkey, Africa (North, Sub-Saharan and Afro-Caribbean), Asia and Latin America represented the most numerous immigrant groups, papers on dietary intake were not available for all populations. Interview-administered FFQ and repeated 24 hour recalls were the most frequently applied instruments. Inclusion of ethnic foods and quantification of specific portion sizes of traditional foods and dishes in assessment tools as well as food composition databases were commonly identified problems. For FFQ, food list elaboration required particular consideration to reflect key ethnic foods and relative contribution to nutrient intake. Extra efforts were observed to overcome cultural barriers to study participation. Evaluating dietary intake of immigrant populations requires special attention to various methodological aspects (sampling, recruiting, instruments used, method of administration, food composition database, acculturation, etc.) so as to adequately address the range of socio-cultural factors inherent in these nutritionally at risk target groups.

Dietary intake: Methods: Ethnic groups: Immigrants: Europe

Immigrants and ethnic minority groups in Europe comprise a noteworthy segment of the population, which has been on the rise over the past decades. A recent review of selected European countries has shown that as a percentage of its total population, France had the largest ethnic minority populations $(21.7 \%)$, followed by The Netherlands (19\%), Germany $(8.8 \%)$, Spain and Denmark (each with $8.5 \%$ ), the UK $(7.9 \%)$ and Italy $(5.6 \%)^{(1)}$. The largest ethnic groups of non-European origin come from the following regions: Turkey; Africa (North and Sub-Saharan); South Asia; Latin America; the Caribbean and East and Southeast Asia ${ }^{(2)}$.

Disparities in health status have been shown for ethnic minority populations when compared with the general population, although differences have also been described between the various immigrant groups $^{(3-5)}$. While the 'healthy migrant' effect, referring to selection bias as those in good health are those who are able to migrate, may be reflected in morbidity and mortality data, clear differences in health status have been observed by geographical variation, ethnicity and acculturation ${ }^{(6,7)}$. Evidence points out that immigrants soon acquire the chronic disease patterns of the host country ${ }^{(8)}$ and even have higher rates of mortality and morbidity linked to certain nutrition-related diseases than the autochthonous population $^{(1,9-12)}$, with rates also being greater than those in their country of origin ${ }^{(13-15)}$.

Compared with the subjects of identical cultural and genetic backgrounds residing in their native countries, Gujarati Indians ${ }^{(15)}$ and Afro-Caribbeans ${ }^{(16)}$ living in the UK had a

Abbreviation: 24HR, 24 hour recalls.

On behalf of EURRECA's RA 1.1 "Intake Methods" members: Serra-Majem L (Coordinator), Cavelaars A, Dhonukshe-Rutten R, Doreste JL, Frost-Andersen L, García-Álvarez A, Glibetic M, De Groot L, Henríquez-Sánchez P, Naska A, Ngo J, Novakovic R, Ortiz-Andrellucchi A, Øverby NC, Gurinovic M, Pijls L, Ranic M, Ribas-Barba L, Ristic-Medic D, Román-Viñas B, Ruprich J, Saavedra-Santana P, Sánchez-Villegas A, Tabacchi G, Tepsic J, Trichopoulou A, van’t Veer P, Vucic V, Wijnhoven TMA.

* Corresponding author: Joy Ngo, fax +34 9340345 43, email nutricom@pcb.ub.cat 
higher prevalence of established CHD risk factors and overweight, respectively. Some affirm that, in accordance with the fetal origins of health theory ${ }^{(17)}$, higher nutritionrelated non-communicable diseases are linked to the more compromised status of ethnic minorities, as many come from countries where nutritional deprivation is still a reality. As such, malnutrition in the womb or during early childhood may be a precursor to pathologies in adulthood, particularly in situations when the standards of living improve ${ }^{(18-20)}$. The process of immigration in and of itself is likely to influence health status with respect to nutrition-related noncommunicable diseases. Predisposition to chronic diseases appears to be amplified by a variety of environmental factors such as diet, low physical activity, immune-inflammatory changes, stress of discrimination, poorer use and lower quality of care ci,18,21). $^{(1,0}$.

There has been growing attention on the influence of acculturation on diet and health in minority populations. Although results may vary due to the fact that the process is complex and multidirectional, acculturation has been linked with suboptimal dietary choices including not breastfeeding, low consumption of fruits and vegetables, and higher intakes of fats and sweetened beverages. In the same way, acculturation has been positively associated with lower physical activity, higher BMI and the likelihood of type 2 diabetes. However, findings have been inconsistent across ethnic groups and subgroups and nutrition outcomes of interest ${ }^{(12,22-25)}$.

Apart from being at increased risk of nutrition-related noncommunicable diseases, ethnic minority groups have also been documented to have inadequate intakes for several nutrients, of which vitamin $\mathrm{D}, \mathrm{Ca}, \mathrm{Fe}$ and folate predominate. Dental caries, parasites and suboptimal birth outcomes have also been cited, among others ${ }^{(1,26-30)}$.

The previously mentioned factors underscore the need for obtaining reliable data on dietary habits and nutrient intake of these nutritionally vulnerable segments of the population. Various challenges to conducting dietary evaluations in ethnic populations include difficulties in assessing individual consumption in those eating from a shared serving dish/pot, understanding of food terms and concepts, scarce information on ethnic dishes and recipes, culture-specific foods and portion sizes, among others ${ }^{(31)}$. Moreover, sound research on nutrient intakes of predominant immigrant groups is relatively limited and is critical for the correct provision of diet counselling, policy development and the implementation of effective interventions. The EURopean micronutrient RECommendations Aligned (EURRECA) network of excellence financed by the European Commission aims to establish Europe-wide scientific consensus on the evidence on which micronutrient recommendations can be used, with special emphasis on nutritionally at risk population groups. A systematic review was conducted within this framework with the purpose of identifying the presently used methods and adaptations required for adequately measuring dietary intake of the principal ethnic minority groups residing in Europe.

\section{Methods and materials}

A comprehensive search strategy was conducted, which included a structured electronic database search, related references and key expert consultations. The final search strategy was derived from consultations with relevant EURRECA partners as well as external sources with key experts in the field coming from academic, research and government institutions. The review focused on adults from the non-European union ethnic groups (European union defined as those comprising the former European union-15: Austria; Belgium; Denmark; Finland; France; Germany; Greece; Ireland; Italy; Luxemburg; Holland; Portugal; Spain; Sweden; the United Kingdom) having the largest populations in Europe. The structured strategy included searching electronic databases (MEDLINE and COCHRANE) with key words and $\mathrm{MeSH}$ (medical subject headings) terms applied as follows:

Block A. Descriptors of immigrants. MeSH term 'immigrant' (includes emigration and immigration, migration, foreigner, resettlement), 'ethnic', MeSH term 'ethnicity' (includes ethnic groups: African Americans, Arabs, Asian Americans, Gypsies, Hispanic Americans, Inuits, Jews; Indian: South, Central and North America and gypsies), MeSH term 'minority group', MeSH term 'culture' (includes acculturation, cross-cultural comparison, cultural diversity, multicultural, cultural characteristics, ethnology), 'refugee', 'internally displaced person'.

Block $B$. Descriptors of dietary intake methods. MeSH term 'nutrition assessment' (includes nutrition survey, diet survey), 'diet assessment', MeSH term 'dietary habits' (includes food habits, diet modification), MeSH term 'diet records' (includes food diary), '24 hour diet* recall', 'food frequency', 'food consumption', 'nutrient intake'.

Block $C$. Descriptors of ethnic group. Include search term for specific ethnic groups: 'Hispanic'; 'Latino'; 'Indian'; 'Pakistan*'; 'Chinese'; 'Moroccan'; 'Turk*'; 'Romani*'; 'multiethnic'; etc.

Search combinations. [Block A] AND [Block B] AND [Block C].

Titles and abstracts were evaluated for inclusion and those without abstracts were not considered. Papers were excluded if the study was published before 1978, not conducted in human subjects, was realised outside of Europe or exclusively in the country of origin, applied rapid evaluation or qualitative methods. However, if data were lacking for a predominant ethnic group, studies from non-European countries with data on the relevant population were included. The scope of the present review was limited to the ethnic groups having the most numerous populations with open access studies. Due to the paucity of studies evaluating intake at the level of micronutrients, the review included research quantifying analysis at the level of macronutrients and food groups. A manual review of related references of selected papers was done to identify additional papers for possible inclusion, as well as realising further consultations with relevant key experts.

\section{Results}

Ethnic groups analysed

Based on the non-European union-15 ethnic groups having the largest populations in Europe, the analysis centred on studies evaluating the following migrant populations: Turks; Africans (North and Sub-Saharan, Afro-Caribbean); 
South Asians; Central and Latin Americans; multiethnic studies. Although Central and Eastern Europeans (Polish, Romanian, Russian, Ukrainian, etc.) represent a substantial number of immigrants, very few studies were available for analysis due to a lack of specific dietary information (i.e. evaluation of health but not nutrition factors, etc.) or language access. East Asians (Chinese, Korean, Japanese) were not included as they had relatively lower numbers than the other groups, and the majority of studies available in English had been conducted outside of Europe (i.e. in North America). However, most ethnic groups in this category were represented in the multi-site studies selected for the multiethnic category (including whites of European or North American background, Blacks of North American or Caribbean background, Asians, South Asians and Hispanics). In contrast, Hispanics, who represent a relatively important segment of the immigrant population, were included as a target population for analysis even though there was a paucity of European data. In this case, non-European immigrant studies focusing on Latin and Central Americans were included if they reflected corresponding ethnic groups residing in Europe. The results of the selection process by ethnic group are illustrated in Table 1, with the $56 \%$ ( $n$ 26) of the studies coming from Europe, as noted in the table.

The electronic database search yielded 2959 citations. Of these, 176 were selected after evaluating the title and abstract according to exclusion criteria, with a final selection of fortysix papers after full review of the papers.

\section{Sampling and recruitment}

Most studies utilised non-probability sampling methods, such as convenience or quota sampling with some using methods of surname analysis from a sampling frame to identify potential subjects. Ethnicity was frequently defined by place of birth, and in countries with longer histories of ethnic diversity, this criterion was applied for parents or grandparents. In the cases having a context where intermarriage was common, criteria included ancestry (i.e. three of four grandparents with origins from the target ethnic group) or self assignment of ethnicity ${ }^{(35,52,53,57,60,63,65-67)}$.

Extra efforts in recruiting and sampling were also observed to overcome cultural barriers to study participation and to ensure representativeness of the sample. Initiatives included the use of bilingual outreach workers, grassroots dissemination and door-to-door canvassing as well as involving key leaders, celebrities, places of worship and local media.

\section{Diet assessment instruments and modes of administration}

Fig. 1 shows the results of methods that were applied in the selected studies so as to evaluate dietary intake of targeted ethnic minority populations. FFQ were the most frequently applied diet assessment instrument used in twenty-seven $(58.7 \%)$ out of the forty-six studies, while ten $(21.7 \%)$ studies used 24 hour recalls (24HR), four $(8.7 \%)$ studies used weighed food records, two $(4.3 \%)$ studies used diet histories and two $(4.3 \%)$ studies used estimated food records. FFQ were frequently used in multiethnic studies $(85 \%$ of the thirteen studies) as well as in African (North and Sub-Saharan, Afro-Caribbean) populations (71\% of fourteen studies). Of the ten studies applying $24 \mathrm{HR}$, this method was carried out in $100 \%$ of the Turkish populations $v .37 \%$ of the Hispanic, $21 \%$ of the African and $7.6 \%$ of the multiethnic groups. Food records applied as the principle diet assessment tool (studies using this method as a form of validation were not considered) were found in only six studies (13\%), and were only applied in studies evaluating the South Asians. This last group demonstrated the greatest variety in terms of the types of dietary assessment instruments administered (Fig. 1).

The main form of administration was by face to face contact as seen in thirty studies $(63 \%) v$. being self-administered in fifteen studies $(32.6 \%)$, the latter applied primarily in South Asian (in the form of estimated food records and weighed food records), followed by multiethnic (in the form of FFQ) studies (Fig. 1). One study used a telephone-based survey

Table 1. Results of systematic search and selection process by ethnic group

\begin{tabular}{lccc}
\hline & & & Papers $(n)$ \\
\cline { 3 - 4 } Ethnic group & Citations & Abstract review + exclusion criteria & Full paper review + exclusion criteria \\
\hline Polish & 28 & 1 & 0 \\
Hispanic (Latin and Central American) & 419 & 27 & $8^{\star}$ \\
Romanian & 10 & 2 & 0 \\
South Asian (Indian, Pakistan, Bangladesh, etc.) & 359 & 35 & $9 \dagger$ \\
North African & 23 & 5 & $4 \ddagger$ \\
Asian (Chinese, Japanese, Korean, etc.) & 551 & 61 & 7 \\
Turkish & 70 & 30 & $2 \S$ \\
African (Afro-Caribbean, Sub-Saharan) & 1355 & 0 & $10 \|$ \\
Russian & 49 & 0 & 0 \\
Bulgarian & 4 & 29 & 0 \\
Multiethnic & 91 & 176 & 139 \\
Total & 2959 & & $46^{\star *}$
\end{tabular}

\footnotetext{
* Data from USA. References ${ }^{(32-39)}$.

+ References $^{(15,21,40-46)}$.

† References (27,47-49)

$\S$ References $^{(27,50)}$.

\| Three studies from Jamaica and South Africa. References ${ }^{(28,51-59)}$.

1. Nine studies from North America. References ${ }^{(30,60-71)}$

** Excluding studies on Asians.
} 

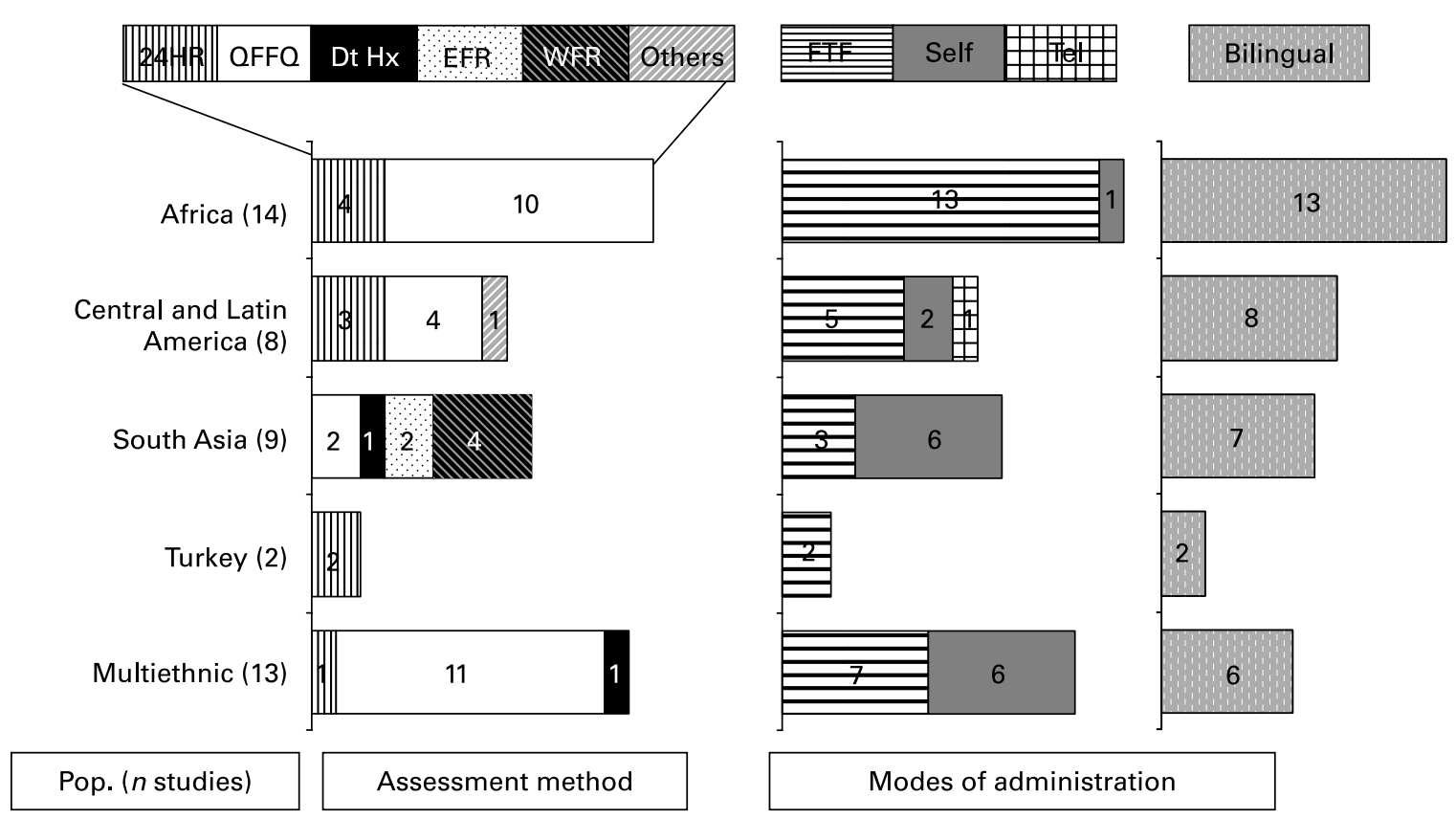

Fig. 1. Diet assessment instruments and modes of administration applied by ethnic group. 24HR, 24 hour recall; QFFQ, quantitative food frequency questionnaire; Dt Hx, diet history; EFR, estimated food record; WFR, weighed food record; FTF, face-to-face interview; Self, self-administered interview; Tel, telephone; Bilingual, bilingual interviewer.

where diet was included as part of a more general health survey ${ }^{(38)}$. Thirty-six studies $(78 \%)$ utilised interviewers that were bilingual or from similar ethnic backgrounds to explain or administer the diet assessment instruments. By ethnic group, the multiethnic studies showed the lowest usage of bilingual or native staff, with only $46 \%$ of the studies providing such interviewers, whereas in other populations, this ranged from 78 to $100 \%$. The lower rate of bilingual/native staff in multiethnic studies was due, in part, to their use of self-administered FFQ, which had been previously translated into the relevant language if required. Some used the same questionnaire for all ethnic groups in the study ${ }^{(66-71)}$, while others had specific FFQ for each minority population $(30,61-63,65)$.

\section{Specific adaptations for dietary assessment of immigrant groups}

Table 2 summarises the key components addressed in the adaptation of study instruments and methodologies to target ethnic populations. Identifying commonly consumed ethnic foods and dishes, portion sizes, recipes and preparation methods was observed and achieved in various ways, with some studies applying several methods to attain a range of objectives (i.e. development of instruments, expanding food composition tables, etc.). Thirty-two (69.6\%) of the studies consulted directly with the target ethnic groups by collecting $24 \mathrm{HR}$ or food records or via in-depth semi-structured interviews or focus groups; nineteen studies (41.3\%) sought advice from experts. In certain studies, specific information on recipes was obtained by referring to cookbooks $(n 9)$ or the Internet $(n$ 1). For those studies using FFQ, twenty-one $(77.8 \%)$ derived information directly from target immigrant groups and/or from experts for the elaboration of food lists and/or for determining weighting factors to assign average nutrient values for multiple foods listed per line item (data not shown).

The quantification of ethnic-specific portion sizes and the inclusion of ethnic foods and traditional dishes in assessment tools as well as food composition databases were the commonly identified problems. For portion size assessment, a known source of measurement error for intake studies in general, thirty-one $(67.4 \%)$ of the studies utilised visual aids, and twenty-three (50\%) applied previously identified serving sizes specific for the target ethnic group instead of commonly used household measures used by the autochthonous population. In reference to food composition databases, thirty-two $(69.6 \%)$ of the studies analysed expanded their database with additional information on specific ethnic foods or dishes. Studies varied in how they obtained this information, ranging from $24 \mathrm{HR}$ interviews, weighed food records or estimated food records and focus groups to expert consultations, cookbooks and internet sources.

Although the target population was consulted to verify content for the development of instruments, only twenty (64.5\%) of the eligible studies conducted feasibility testing to assess comprehensibility, ease of administration, completeness and appropriateness with the ethnic groups in question (data not shown). Even fewer studies ( $5,17.2 \%$ ) complied with the recommended process of forward and back-translation, when applicable (data not shown). Thirty-two (86.5\%) of the studies included some measure of acculturation, ranging from the length of time in the host country to primary language spoken, self-identified ethnicity or degree of integration in social circles. Twenty-two $(77.4 \%)$ of identified papers had conducted correlation (validation) studies for the diet assessment instrument applied (FFQ validated by weighed food record or 24HR). The variation in methods applied and nutrients evaluated in these correlation studies make comparability difficult and is beyond the scope of the present review. 


\section{Discussion}

The present review confirms that the evaluation of dietary intakes in immigrant populations requires adaptations that not only take into account the intake assessment tools but also study methodologies such as the sampling frame and recruitment measures.

The studies included in the present review used a variety of methods to ensure adequate response rates, while obtaining the most representative samples possible. To reduce obstacles to participation, such as illiteracy, language barriers, apprehension, distrust, etc., recruitment methods required extra efforts and could also vary between ethnic groups. Outreach staff was frequently of the same ethnic background and the involvement of key community leaders was solicited. Such initiatives are often required to overcome typically low response rates commonly observed with immigrant populations $(40,43,64,71)$.

Another factor relating to the importance of appropriate sampling and recruitment methods is that within the same ethnic group, socio-economic and geographical differences can also impact on representative participation and thus study results ${ }^{(38,43,45,58)}$. A clear example of this is in Kassam-Khamis's ${ }^{(42,43)}$ work that shows important variations in South Asian dietary habits as they encompass Indian (Gujarati Hindus, Punjabi Sikhs), Pakistani, Bangladeshi and East African Ismailis and as such, reflect not only religious but also geographical diversity. They even went so far as to verify the food frequency checklist under development for Pakistani subjects in three other locations as the sample from the original site (Southall, London) did not represent the majority of Pakistanis living in Britain ${ }^{(43)}$. Finally, if sampling is not directed or purposive, those subjects participating in a study may only reflect more educated or motivated individuals, and thus often under-represent the 'true' target population $^{(72,73)}$

The greater utilisation of FFQ seen in the present review may be due to their use in multiethnic (mostly North American) and African (mostly British) studies, whose subjects from varying ethnic backgrounds had longer times of residence in the host country. Some showed mean lengths of residence ranging from 18 to 25 years (data not shown) ${ }^{(30,57)}$. Studies whose subjects were recent migrants or with limited literacy or language skills relied on the use of (repeated) 24HR for diet assessment. The extensive utilisation of previously trained ethnic interviewers or interpreters shows the importance of not only knowing the target population's language but also the specific details and context of their food culture, which enhances the accuracy of dietary data collection ${ }^{(33,74)}$.

For the transformation of dietary information into quantified nutrient estimates, the food composition database should include commonly consumed ethnic foods and dishes, of which data are often lacking. Detailed recipe information must be obtained to determine ingredients and preparation techniques. Acquiring recipes from cookbooks or the internet is unadvisable as the source of information may not reflect procedures, ingredients and quantities actually used. One study conducting correlation analysis for content validation had to readjust recipes and weights for foods in FFQ line items as $24 \mathrm{HR}$ data revealed that subjects' recipes were much simpler than the internet recipes initially utilised ${ }^{(39)}$. 
The present review highlights the need for further rigour in this area as fourteen (31\%) of the studies reviewed had incomplete descriptions of the methodology applied to adapt food composition databases. Apart from the need to compile recipes for commonly consumed ethnic dishes, information on the effects of prolonged cooking and reheating practices is also an issue to consider ${ }^{(11)}$.

Content validation is a critical step for determining the kinds of food eaten, frequency of consumption, meal patterns, preparation of ethnic dishes, ethnic-specific portions and household measures and whether terminology used is familiar to the study population. Similar foods may be called by different names depending on the geographical origins of the target ethnic group (i.e. beans/pulses have at least four different Spanish translations depending on geographical origin). Moreover, the difficulty of conceptualising food intake according to an unfamiliar classification system (i.e. food groups based on macronutrient content) needs to be addressed as how foods are grouped may contribute significantly to the classification of individuals based on their intake ${ }^{(75)}$. This can be addressed by probing into culture-specific food group categories with the target populations. Other methods have grouped foods by those most consumed to those least consumed ${ }^{(55)}$ or applied a classification system consisting of main dishes, side dishes, snacks and desserts ${ }^{(76)}$.

Furthermore, verifying information with the target group is needed to identify variation in recipes and cooking practices, among other factors ${ }^{(11,31)}$. For example, substantial recipe variation has been documented among South Asians ${ }^{(40,43,77)}$ due to differences in geographical origin, ingredient availability or method of cooking. As a result, the food list and food composition database developed in KassamKhamis's ${ }^{(43,45)}$ work included forty-six composite dishes encompassing South Asian traditional dishes for meat, vegetables and pulses. Moreover, for thirty-one of these forty-six dishes, three to four different recipes per dish were included and data used for analysis was based on the subject's region of origin, religious background as well as the type of fat used (i.e. ghee, vegetable oil), and whether onions or tomatoes were included. In another study, content validation with the target populations revealed discrepancies in the weights of fried plantain portions actually used (average of $30 \mathrm{~g}) v$. what was contained in the food composition database $(74.5 \mathrm{~g})^{(39)}$. Although expert consultation may be suitable for verifying certain information, it is recommended to directly corroborate content with the target population, and some studies have combined the two approaches. As such, merely 'adding' new items to a previously validated instrument with minimal content validation for a certain ethnic group is not recommended and may jeopardise the validity of study results.

With respect to portion sizes, the use of visual aids is critical in immigrant populations as the concept of servings may not even exist for certain cultures, particularly those who eat from a communal serving dish. Two- and three-dimensional food photographs as well as three-dimensional food models appear to be more effective than only using common household measures, irrespective of mode (telephone $v$. faceto-face interview), although interviewer skills can also be a mediating factor ${ }^{(74,78-80)}$. For those studies utilising quantitative FFQ, apart from the use of visual aids, the variety of methods for estimating usual portion size (i.e. fixed $v$. open ended) shows a lack of consensus of which is the preferred mode to apply in immigrant populations. A study in Puerto Rican subjects showed improvement in correlation coefficients by adapting host country standard portions to ethnic-specific portions or by applying open-ended methods ${ }^{(39)}$. In reference to estimating portions in persons who shared a communal dish, no methodological data were found that addressed this in detail. However, in the majority of these populations, $24 \mathrm{HR}$ interviews were conducted primarily in the subject's home, which helped to determine the household measures commonly used, apart from the use of visual aids. Other approaches included determining the amount consumed as a proportion of the total amount of food prepared, subtracting leftovers and dividing by the number of people in the household, taking into account and adjusting for children. (Personal communication M Nicolaou, L Ribas-Barba, unpublished results).

Apart from using visual aids to determine portions, utilising ethnic-specific portion sizes was another relevant issue as only $50 \%$ of the studies included in the present review explained their methods for this adjustment, and usual servings can vary between different minority groups (Fig. 2). A 3-fold difference was observed for a serving of West Indian soup in comparison with the standard portion size in the UK (data not shown). One study using multiple 24HR telephone recalls for FFQ validation previously determined average weights of commonly consumed ethnic dishes in tablespoons and serving spoons, which were then applied in the telephone recalls to estimate portion sizes as these household measures were those utilised by the target population ${ }^{(45)}$. Culturally appropriate portion size representation is critical to avoid under- or overreporting, which can be influenced by cultural factors such as social desirability and status foods ${ }^{(81,82)}$ Under-reporting is of special concern as it is associated with heavier body weight, and high overweight/obesity rates are frequently seen in ethnic and low-income $\operatorname{groups}(12,18,26)$. Mennen ${ }^{(56,83)}$ found that in four populations of African originrural and urban Cameroon, Jamaica and African Caribbeans in the United Kingdom, those in the United Kingdom (who also had higher percentages of overweight) had the highest frequency of underreporting (EI/BMRest of 1.15) with a mean percentage of $6,4.5,21.5$ and $33.5 \%$, respectively.

One of the areas where further rigour is needed is in the process of translating assessment instruments. The use of forward and back-translation is a widely used method in cross-cultural research, and when combined with additional bilingual and

\begin{tabular}{|l|c|c|c|}
\hline Food & $\begin{array}{c}\text { West } \\
\text { Indian (UK) }^{\mathrm{a}}\end{array}$ & $\begin{array}{c}\text { Puerto } \\
\text { Rican (USA) }^{\mathrm{b}}\end{array}$ & $\begin{array}{c}\text { White } \\
\text { American }^{\mathrm{b}}\end{array}$ \\
\hline Rice, plain & 176 & 231 & 128 \\
\hline Banana & 100 & 121 & 94 \\
\hline Non-citrus juice & 148 & 222 & 169 \\
\hline Hamburger & 75 & 251 & 160 \\
\hline Fried chicken & 113 & 101 & 63 \\
\hline Soup & 542 & 406 & 305 \\
\hline Dark bread & 74 & 42 & 38 \\
\hline Cake, cookie & 107 & 68 & 52 \\
\hline
\end{tabular}

Fig. 2. Mean portion sizes (g) for selected foods and ethnic groups. ${ }^{a}$ Sharma $2002^{(59)}$. ' Tucker $1998^{(39)}$. 
monolingual post-translation testing, it is considered as the most complete instrument translation process ${ }^{(84,85)}$. Even though various methodologies can be applied to achieve this and there is presently no gold standard established, the results of the present review showed poor compliance and/or documentation ( $83 \%$ of twenty-nine studies utilising translated materials lacked this information) of meeting the minimum standard of back-translation with posterior monolingual (target population) testing. Better compliance was seen in the feasibility testing of instruments with target ethnic groups (only $35 \%$ of thirty-one applicable studies lacked this documentation). However, this highlights the need for increased efforts to assure that concepts and terminology are understood. Apart from the diversity of terms used for similar foods, culture-based perceptions on food groupings and portions may not coincide with the host country's classification or even in the wording of questions. For example, screening for rice consumption requires additional clarification in those cultures habitually consuming rice in combination with pulses. 'How often do you eat rice?' for Latino and AfroCaribbeans would be better stated '....as plain rice or as rice and beans/peas and rice?', since each phrase has a different culinary connotation ${ }^{(32,58)}$.

Finally, the present review shows that further research is needed to fill knowledge gaps for certain immigrant groups (Central and Eastern Europe), with special emphasis on the Roma population as they are a markedly marginalised and hard-to-reach group as well as internally displaced persons and refugees ${ }^{(86,87)}$. European data on Central and South America as well as East and Southeast Asia (Chinese, Vietnamese, Filipinos) are also lacking as well as well-defined acculturation scales.

\section{Conclusion}

Evaluating dietary intake of immigrant populations requires that special attention be paid to various methodological aspects (sampling and recruiting, instruments used, method of administration, food composition database, acculturation, among others) so as to adequately address the ample range of socio-cultural factors inherent in these nutritionally at risk target groups.

\section{Acknowledgements}

The studies reported herein have been carried out within the EURRECA network of excellence (www.eurreca.org), financially supported by the Commission of the European Communities, specific Research, Technology and Development (RTD) Programme Quality of Life and Management of Living Resources, within the Sixth Framework Programme, contract no. 036196. The present report does not necessarily reflect the Commission's views or its future policy in this area. J. N. developed and carried out the search strategy, undertook analysis and wrote the first draft of the paper. M. G. participated in the planning of the search strategy and commented on following drafts of the paper. L. F.-A. commented on drafts of the paper. L. S.-M. participated in the planning of the strategy, directed and supervised the work and commented on all drafts of the paper. Additional support from Dr. Margaret Ashwell and Dr. Janet Lambert in reviewing concepts and contents is gratefully acknowledged. The authors have no conflict of interests to report.

\section{References}

1. Gilbert PA \& Khokhar S (2008) Changing dietary habits of ethnic groups in Europe and implications for health. Nutr Rev 66, 203-215.

2. Migration Policy Institute, available at: http://www.migration policy.org/research/europe.php (accessed August 2008).

3. Bhopal R, Unwin N, White M, et al. (1999) Heterogeneity of coronary heart disease risk factors in Indian, Pakistani, Bangladeshi, and European origin populations: cross sectional study. BMJ 319, 215-220.

4. Glenday K, Kumar BN, Tverdal A, et al. (2006) Cardiovascular disease risk factors among five major ethnic groups in Oslo, Norway: The Oslo Immigrant Health Study. Eur J Cardiovasc Prev Rehabil 13, 348-355.

5. Kumar BN, Meyer HE, Wandel M, et al. (2006) Ethnic differences in obesity among immigrants from developing countries, in Oslo, Norway. Int J Obes (Lond) 30, 684-690.

6. Hyman I (2007) Immigration and health: reviewing evidence of the healthy immigrant effect in Canada. CERIS (Centre of Excellence for Research on Immigration and Settlement) Working Paper no. 55. [MJ Doucet, editor]. Toronto: CERIS. Available at: http://ceris.metropolis.net/Virtual\%20Library/ WKPP\%20List/WKPP2007/CWP55.pdf

7. Razum O, Zeeb H, Akgün HS, et al. (1998) Low overall mortality of Turkish residents in Germany persists and extends into a second generation: merely a healthy migrant effect? Trop Med Int Health 3, 297-303.

8. Gushulak BD \& MacPherson DW (2006) The basic principles of migration health: population mobility and gaps in disease prevalence. Emerg Themes Epidemiol, available at: http://www.ete-online.com/content/3/1/3

9. Bollini P \& Siem H (1995) No real progress towards equity: health of migrants and ethnic minorities on the eve of the year 2000. Soc Sci Med 41, 819-828.

10. Bos V, Kunst AE, Keij-Deerenberg IM, et al. (2004) Ethnic inequalities in age- and cause-specific mortality in The Netherlands. Int J Epidemiol 33, 1112-1119.

11. Church S, Gilbert P \& Khokhar S (2006) Ethnic Groups and Foods in Europe. Synthesis report no. 3. London: Eurofir Project Management Office/British Nutrition Foundation.

12. Darmon N \& Khlat M (2001) An overview of the health status of migrants in France, in relation to their dietary practices. Public Health Nutr 4, 163-172.

13. Bjerregaard P, Jorgensen ME, Lumholt P, et al. (2002) Higher blood pressure among Inuit migrants in Denmark than among the Inuit in Greenland. J Epidemiol Community Health 56, 279-284.

14. Gadd M, Johansson SE, Sundquist J, et al. (2006) Are there differences in all-cause and coronary heart disease mortality between immigrants in Sweden and in their country of birth? A followup study of total populations. BMC Public Health 6, 102.

15. Patel JV, Vyas A, Cruickshank JK, et al. (2006) Impact of migration on coronary heart disease risk factors: comparison of Gujaratis in Britain and their contemporaries in villages of origin in India. Atherosclerosis 185, 297-306.

16. Jackson M, Walker S, Cruickshank JK, et al. (2007) Diet and overweight and obesity in populations of African origin: Cameroon, Jamaica and the UK. Public Health Nutr 10, 122-130.

17. Barker DJP (1994) Mothers, Babies and Disease in Later Life. London: BMJ Publishing Group.

18. Landman L \& Cruickshank JK (2001) A review of ethnicity, health and nutrition related diseases in relation to migration in the United Kingdom. Pub Health Nutr 4, 647-657. 
19. Stein AD, Zybert PA, van der Pal-de Bruin K, et al. (2006) Exposure to famine during gestation, size at birth, and blood pressure at age 59 y: evidence from the Dutch Famine. Eur $J$ Epidemiol 21, 759-765.

20. Mellin-Olsen T \& Wandel M (2005) Changes in food habits among Pakistani immigrant women in Oslo, Norway. Ethn Health 10, 311-339.

21. Wandel M, Råberg M, Kumar B, et al. (2008) Changes in food habits after migration among South Asians settled in Oslo: the effect of demographic, socio-economic and integration factors. Appetite 50, 376-385.

22. Satia-Abouta J, Patterson R, Neuhouser ML, et al. (2002) Dietary acculturation: applications to nutrition research and dietetics. J Am Diet Assoc 102, 1105-1118.

23. Mejean C, Traissac P, Eymard-Duvernay S, et al. (2007) Diet quality of North African migrants in France partly explains their lower prevalence of diet-related chronic conditions relative to their native French Peers. J. Nutr 137, 2106-2113.

24. Gordon-Larsen P, Mullan-Harris K, Ward DS, et al. (2003) Acculturation and overweight-related behaviors among Hispanic immigrants to the US: The National Longitudinal Study of Adolescent Health. Soc Sci Med 57, 2023-2034.

25. Pérez-Escamilla R \& Putnik P (2007) The role of acculturation in nutrition, lifestyle, and incidence of type 2 diabetes among Latinos. J Nutr 137, 860-870.

26. Brussaard JH, van Erp-Baart MA, Brants HAM, et al. (2001) Nutrition and health among migrants in The Netherlands. Public Health Nutr 4, 659-664.

27. Palsma AH, Nicolaou M, van Dam RM, et al. (2006) De voeding van Turkse en Marokkaanse Nederlanders in de leeftijd van 18 - 30 jaar (Food consumption of Turkish and Moroccans in The Netherlands between the age of 18-30 years). Tsg jaargang 84, 415-422, (In Dutch).

28. van Dam RM, Nicolaou M \& Stronks K (2005) Voedingspatroon van Surinaamse Amsterdammers in kaart gebracht (Study of the diet of Surinamese Amsterdammers). Ned Tijdschr Diet 60, 98-102, (In Dutch).

29. Rees GA, Doyle W, Srivastava A, et al. (2005) The nutrient intakes of mothers of low birth weight babies - a comparison of ethnic groups in East London, UK. Matern Child Nutr 1, 91-99.

30. Vyas A, Greenhalgh A, Cade J, et al. (2003) Nutrient intakes of an adult Pakistani, European and African-Caribbean community in inner city Britain. J Hum Nutr Diet 16, 327-337.

31. Khokhar S, Pandor A \& Cade J (2001) Eating Habits: Food Preparation and Serving Practices in Ethnic Populations: Critical Review and Assessment of Dietary Survey Requirements. Report to the Food Standards Agency, London, UK. FSA Project: N08015.

32. Block G, Norris JC, Mandel RM, et al. (1995) Sources of energy and six nutrients in diets of low-income Hispanic-American women and their children: quantitative data from HHANES, 1982-1984. J Am Diet Assoc 95, 195-208.

33. Block G, Wakimoto P, Jensen C, et al. (2006) Validation of a food frequency questionnaire for Hispanics. Prev Chronic Dis 3, (Serial online), available at: http://www.cdc.gov/pcd/issues/ 2006/jul/05_0219.htm (accessed September 2008).

34. Kristal AR, Shattuck AL \& Patterson RE (1999) Differences in fat-related dietary patterns between black, Hispanic and White women: results from the Women's Health Trial Feasibility Study in Minority Populations. Public Health Nutr 2, 253-262.

35. Loria CM, Bush TL, Carroll MD, et al. (1995) Macronutrient intakes among adult Hispanics: a comparison of Mexican Americans, Cuban Americans, and mainland Puerto Ricans. Am J Public Health 85, 684-689.

36. Nath SD \& Huffman FG (2005) Validation of a semiquantitative food frequency questionnaire to assess energy and macronutrient intakes of Cuban Americans. Int J Food Sci Nutr 56, 309-314.

37. Neuhouser ML, Thompson B, Coronado GD, et al. (2004) Higher fat intake and lower fruit and vegetables intakes are associated with greater acculturation among Mexicans living in Washington State. J Am Diet Assoc 104, 51-57.

38. Ramirez AG, Suarez L, Chalela P, et al. (2004) Cancer risk factors among men of diverse Hispanic or Latino origins. Prev Med 39, 263-269.

39. Tucker KL, Bianchi LA, Maras J, et al. (1998) Adaptation of a food frequency questionnaire to assess diets of Puerto Rican and non-Hispanic adults. Am J Epidemiol 148, 507-518.

40. Anderson AS, Bush H, Lean M, et al. (2005) Evolution of atherogenic diets in South Asian and Italian women after migration to a higher risk region. J Hum Nutr Diet 18, 33-43.

41. Eaton PM, Wharton PA \& Wharton BA (1984) Nutrient intake of pregnant Asian women at Sorrento Maternity Hospital, Birmingham. Br J Nutr 52, 457-468.

42. Kassam-Khamis T, Nanchachal K, Mangtani P, et al. (1999) Development of an interview-administered food frequency questionnaire for use amongst women of South Asian ethnic origin in Britain. J Hum Nutr Diet 12, 7-19.

43. Kassam-Khamis T, Judd PA \& Thomas JE (2000) Frequency of consumption and nutrient composition of composite dishes commonly consumed in the UK by South Asian Muslims originating from Bangladesh, Pakistan and East Africa (Ismailis). J Hum Nutr Diet 13, 185-196.

44. Lovegrove JA, Lovegrove SS, Lesauvage SVM, et al. (2004) Moderate fish-oil supplementation reverses low-platelet, longchain $n-3$ polyunsaturated fatty acid status and reduces plasma triacylglycerol concentrations in British Indo-Asians. Am J Clin Nutr 79, 974-982.

45. Sevak L, Mangtani P, McCormack V, et al. (2004) Validation of a food frequency questionnaire to assess macro- and micronutrient intake among South Asians in the United Kingdom. Eur J Nutr 43, 160-168.

46. Wharton PA, Eaton PM \& Wharton BA (1984) Subethnic variation in the diets of Moslem, Sikh and Hindu pregnant women at Sorrento Maternity Hospital, Birmingham. Br J Nutr 52, 469-476.

47. Mejean C, Traissac P, Eymard-Duvernay S, et al. (2007) Diet quality of North African migrants in France partly explains their lower prevalence of diet-related chronic conditions relative to their native French peers. J Nutr 137, 2106-2113.

48. Montoya Sáez PP, Torres Cantero AM \& Torija Isasa ME (2001) Nutrition among Moroccan immigrants in the community of Madrid: factors affecting the choice of food. Aten Primaria 27, 264-270, (In Spanish).

49. van Erp-Baart MA, Westenbrink S, Hulshof KF, et al. (2001) Assessment of dietary intake among Moroccan women and Surinam men. Ethn Health 6, 145-154.

50. Daryani A, Basu S, Becker W, et al. (2007) Antioxidant intake, oxidative stress and inflammation among immigrant women from the Middle East living in Sweden: associations with cardiovascular risk factors. Nutr Metab Cardiovasc Dis 17, 748-756.

51. Gil A, Vioque J \& Torija E (2005) Usual diet in Bubis, a rural immigrant population of African origin in Madrid. J Hum Nutr Diet 18, 25-32.

52. Jackson M, Walker S, Cade J, et al. (2001) Reproducibility and validity of a quantitative food-frequency questionnaire among Jamaicans of African origin. Public Health Nutr 4, 971-980.

53. Jackson M, Walker S, Cruickshank JK, et al. (2007) Diet and overweight and obesity in populations of African origin: Cameroon, Jamaica and the UK. Public Health Nutr 10, 122-130.

54. MacIntyre UE, Venter CS \& Vorster HH (2001) A culturesensitive quantitative food frequency questionnaire used in an African population: 1. Development and reproducibility. Public Health Nutr 4, 53-62. 
55. MacIntyre UE, Venter CS \& Vorster HH (2001) A culturesensitive quantitative food frequency questionnaire used in an African population: 2. Relative validation by 7 -day weighted records and biomarkers. Public Health Nutr 4, 63-71.

56. Mennen LI, Jackson M, Sharma S, et al. (2001) Habitual diet in four populations of African origin: a descriptive paper on nutrient intakes in rural and urban Cameroon, Jamaica and Caribbean migrants in Britain. Public Health Nutr 4, 765-772.

57. Sharma S, Cade J, Riste L, et al. (1999) Nutrient intake trends among African-Caribbeans in Britain: a migrant population and its second generation. Public Health Nutr 2, 469-476.

58. Sharma S \& Cruickshank JK (2001) Cultural differences in assessing dietary intake and providing relevant dietary information to British African-Caribbean populations. J Hum Nutr Diet 14, 449-456.

59. Sharma S, Cade J, Landman J, et al. (2002) Assessing the diet of the British African-Caribbean population: frequency of consumption of foods and food portion sizes. Int J Food Sci Nutr 53, 439-444.

60. Arab L, Carriquiry A, Steck-Scott S, et al. (2003) Ethnic differences in the nutrient intake adequacy of premenopausal US women: results from the Third National Health Examination Survey. J Am Diet Assoc 103, 1008-1014.

61. Block G, Mandel R \& Gold E (2004) On food frequency questionnaires: the contribution of open-ended questions and questions on ethnic foods. Epidemiology 15, 216-221.

62. Gold EB, Bair Y, Block G, et al. (2007) Diet and lifestyle factors associated with premenstrual symptoms in a racially diverse community sample: Study of Women's Health Across the Nation (SWAN). J Womens Health (Larchmt) 16, 641-656.

63. Heald AH, Cade JE, Cruickshank JK, et al. (2003) The influence of dietary intake on the insulin-like growth factor (IGF) system across three ethnic groups: a population-based study. Public Health Nutr 6, 175-180.

64. Holvik K, Meyer HE, Haug E, et al. (2005) Prevalence and predictors of vitamin $\mathrm{D}$ deficiency in five immigrant groups living in Oslo, Norway: The Oslo Immigrant Health Study. Eur J Clin Nutr 59, 57-63.

65. Kelemen LE, Anand SS, Vuksan V, et al. (2003) Development and evaluation of cultural food frequency questionnaires for South Asians, Chinese, and Europeans in North America. $J$ Am Diet Assoc 103, 1178-1184.

66. Kolonel LN, Henderson BE, Hankin JH, et al. (2000) A multiethnic cohort in Hawaii and Los Angeles: baseline characteristics. Am J Epidemiol 151, 346-357.

67. Kristal AR, Feng Z, Coates RJ, et al. (1997) Associations of race/ethnicity, education, and dietary intervention with the validity and reliability of a food frequency questionnaire: The Women's Health Trial Feasibility Study in Minority Populations. Am J Epidemiol 146, 856-869.

68. Mayer-Davis EJ, Vitolins MZ, Carmichael SL, et al. (1999) Validity and reproducibility of a food frequency interview in a multi-cultural epidemiology study. Ann Epidemiol 9, 314-324.

69. Nettleton JA, Steffen LM, Mayer-Davis EJ, et al. (2006) Dietary patterns are associated with biochemical markers of inflammation and endothelial activation in the multi-ethnic study of atherosclerosis (MESA). Am J Clin Nutr 83, 1369-1379.

70. Shahar D, Shai I, Vardi H, et al. (2003) Development of a semiquantitative food frequency questionnaire (FFQ) to assess dietary intake of multiethnic populations. Eur J Epidemiol 18, 855-861.

71. Stram DO, Hankin JH, Wilkens LR, et al. (2000) Calibration of the dietary questionnaire for a multiethnic cohort in Hawaii and Los Angeles. Am J Epidemiol 151, 358-370.

72. Hussain-Gambles M, Leese B, Atkin K, et al. (2004) Involving South Asian patients in clinical trials. Health Technol Assess 8 , $42,1-128$

73. Lewis CE, George V, Fouad M, et al. (1998) Recruitment strategies in the women's health trial: feasibility study in minority populations. WHT: FSMP Investigators Group. Women's Health Trial: feasibility study in minority populations. Control Clin Trials 19, 461-476.

74. Thompson FE \& Subar AF (2008) Dietary assessment methodology. In Nutrition in the Prevention and Treatment of Disease, 2nd ed., pp. 3-39 [A Coulston and C Boushey, editors]. London: Elsevier.

75. Coates RJ \& Monteilh CP (1997) Assessments of foodfrequency questionnaires in minority populations. Am J Clin Nutr 65, Suppl, 1108S-1115S.

76. Teufel NI (1997) Development of culturally competent food-frequency questionnaires. Am J Clin Nutr 65, Suppl, 1173S-1178S

77. Wharton PA, Eaton PM \& Day KC (1983) Sorrento Asian food tables: food tables, recipes and customs of mothers attending Sorrento maternity hospital, Birmingham, England. Hum Nutr Appl Nutr 37A, 378-402.

78. Godwin SL, Chambers E \& Cleveland L (2004) Accuracy of reporting dietary intake using various portion-size aids inperson and via telephone. J Am Diet Assoc 104, 585-594

79. Turconi G, Guarcello M, Gigli-Berzolari F, et al. (2005) An evaluation of a colour food photography atlas as a tool for quantifying food portion size in epidemiological dietary surveys. Eur J Clin Nutr 59, 923-931.

80. Venter CS, MacIntyre UE \& Vorster HH (2000) The development and testing of a food portion photograph book for use in an African population. J Hum Nutr Diet 13, 205-218.

81. Mendez MA, Wynter S, Wilks R, et al. (2004) Under- and overreporting of energy is related to obesity, lifestyle factors and food group intakes in Jamaican adults. Public Health Nutr $\mathbf{7}$, 9-19.

82. Harrison GG (2004) Methodologic considerations in descriptive food- consumption surveys in developing countries. Proceedings of the Workshop on Food- Consumption Surveys in Developing Countries. Food Nutr Bull 25, 415-419.

83. Mennen LI, Jackson M, Cade J, et al. (2000) Underreporting of energy intake in four populations of African origin. Int $J$ Obes Relat Metab Disord 24, 882-887.

84. Cha ES, Kim KH \& Erlen JA (2007) Translation of scales in cross-cultural research: issues and techniques. $J$ Adv Nurs $\mathbf{5 8}$, 386-395.

85. Maneesriwongul W \& Dixon JK (2004) Instrument translation process: a methods review. $J$ Adv Nurs 48, 175-186.

86. Pavlovic M (2003) Eating Patterns and Nutrient Intake of Internally Displaced Persons (IDPs) in FRY, Report of the UNHCR and WFP Joint Assessment Mission, IDPs in Serbia and Montenegro, UNHCR.

87. Lo Castro L, Guest P, Pavlovic M, et al. (2003) Report of the UNHCR and WFP 2003. Joint Assessment Mission, for refugees in Serbia and Montenegro, Geneva, Rome, Belgrade. 